\title{
Study of Propagation Mechanisms in Dynamical Railway Environment to Reduce Computation Time of 3D Ray Tracing Simulator
}

\author{
Siham Hairoud, ${ }^{1,2}$ Pierre Combeau, ${ }^{1}$ Yannis Pousset, ${ }^{1}$ Yann Cocheril, ${ }^{2}$ \\ Marion Berbineau, ${ }^{2}$ and Rodolphe Vauzelle ${ }^{1}$ \\ ${ }^{1}$ XLIM UMR CNRS 7252, SIC Department, University of Poitiers, Bât. SP2MI, Téléport 2, Boulevard Marie et Pierre Curie, BP 30179 , \\ 86962 Futuroscope Cedex, France \\ ${ }^{2}$ University of Lille Nord de France, and IFSTTAR, LEOST, 59650 Villeneuve D’Ascq, France
}

Correspondence should be addressed to Pierre Combeau; pierre.combeau@xlim.fr

Received 22 January 2013; Revised 4 April 2013; Accepted 5 April 2013

Academic Editor: Hon Tat Hui

Copyright (C) 2013 Siham Hairoud et al. This is an open access article distributed under the Creative Commons Attribution License, which permits unrestricted use, distribution, and reproduction in any medium, provided the original work is properly cited.

In order to better assess the behaviours of the propagation channel in a confined environment such as a railway tunnel for subway application, we present an optimization method for a deterministic channel simulator based on 3D ray tracing associated to the geometrical optics laws and the uniform theory of diffraction. This tool requires a detailed description of the environment. Thus, the complexity of this model is directly bound to the complexity of the environment and specifically to the number of facets that compose it. In this paper, we propose an algorithm to identify facets that have no significant impact on the wave propagation. This allows us to simplify the description of the geometry of the modelled environment by removing them and by this way, to reduce the complexity of our model and therefore its computation time. A comparative study between full and simplified environment is led and shows the impact of this proposed method on the characteristic parameters of the propagation channel. Thus computation time obtained from the simplified environment is 6 times lower than the one of the full model without significant degradation of simulation accuracy.

\section{Introduction}

Modern subways and particularly driverless systems use Communications Based Train Control (CBTC) systems that rely on continuous radio communications in the $2-6 \mathrm{GHz}$ band. These systems are based on COTS (Component Of The Shell) such as IEEE 802.11x modems on which proprietary modifications have been implemented in order to guaranty Key Performance Indicators (KPIs) related to availability, robustness of the radio links, end to end Quality of Services (QoS), handover duration, latency, and so forth. Today, MIMO (Multiple Input-Multiple Output) techniques have proven their ability to increase robustness or data rate and are implemented in emerging standards such as IEEE 802.11n, WiMAX, and LTE. Nevertheless, important degradation of performance can occur in subway tunnels when there is spatial correlation in the channel $[1,2]$. In order to develop new
MIMO algorithms able to cope with dynamic behavior of the channel (correlation but also moving trains), it is necessary to have a clear idea about the propagation channel mechanisms. This can be done by channel sounding campaigns or realistic propagation models.

In the literature, we find a lot of propagation models connected to confined environments such as tunnels. They can be split into two main families of propagation models, that is, those based on measurements and on the Maxwell equations.

For the measurement based models, the most used ones are [3-5]. These models are usually very fast in terms of computation time but are not site specific and do not take into account the dynamical scenario and the presence of masking train. Concerning the models based on Maxwell's equations, we can distinguish the rigorous models, 
which consist in a numerical resolution of the equations [69], the modal theory based models [10-15], and the frequency asymptotic models based on ray concept [16-18]. Although the rigorous models can be theoretically used whatever the tunnel environments, the necessary spatial discretization (about a tenth of wavelength) makes impossible their use in real tunnels in regard to their dimensions and the presence of trains inside the tunnels. On the other hand, the modal theory only treats canonical tunnel assimilated to oversized dielectric waveguides. Furthermore, both theories have not been used in the case of dynamical scenarios with masked trains. In the specific case of dynamical environments, few investigations have been carried out such as in [19] where the authors deal with high-speed railway tunnels $(300 \mathrm{~km} / \mathrm{h})$ whereas in our paper we are interested in metro tunnel in which trains' speed is less than $80 \mathrm{~km} / \mathrm{h}$. Furthermore, they statistically characterize from measurements campaign the propagation by a path loss exponent, the standard deviation of the shadowing, and the distribution of the fast fading (Rice or Rayleigh). Finally, the ray-based models are able to treat tunnel environments of any shape, even in dynamical scenario [20] with trains moving inside the tunnel between the antennas. In addition, it had been explored extensively by Maurer and coworkers to study vehicle-to-vehicle scenario $[21,22]$ and shown to agree well with measurements [2328]. However, the computational complexity of this method is directly related to the number of environment scatters and electromagnetic interactions. There are some acceleration techniques of the computation time in literature but they focus on preprocessing of the database $[29,30]$ or some other techniques using the genetic algorithm [31], visibility tree algorithms [32, 33], the efficient data structures [34], or a fast 3D method of regions algorithm [35].

In this paper, we propose a novel optimization technique to reduce the computation time of a $3 \mathrm{D}$ ray tracing based simulator that presents the advantage to be complementary in regards of previous methods. Since computation time of such a simulator is mainly connected to the number of facets composing the environment, the idea developed in this paper consists in trying to know if it is possible to reduce it while keeping simulations of a level of acceptable accuracy. To answer this question, we have in a first time to identify the contribution degree of each facet on the wave propagation. In a second time, we simplify the considered environment by removing the identified nonsignificant facets. The aim of our method is, from this simplified environment, to provide estimated power and channel characteristic parameters close to those obtained with a full environment modelling, but in a fewer computation time.

So the paper is organized as follows. Section 2 briefly describes the outline of our radio wave propagation simulator and our characterization tool used to respectively predict the radio wave propagation behaviour and to extract the characteristic parameters of the propagation channel. In Section 3 we describe the proposed method to identify the nonsignificant scatters. In Section 4 we apply this last one on a realistic scenario that drives to a simplification of the environment by eliminating the nonsignificant scatters. Thus, we show the weak impact of these geometrical simplifications on the narrow and wide band channel parameters. Then, in Section 5 we introduce an engineering method based on simple geometrical criterion to simplify the description of underground and confined environments like subway tunnels. This one is validated on a last scenario. Finally, the conclusion in Section 6 wraps up this paper.

\section{Modeling and Characterizing the Mobile Radio Channel}

In this section, we quickly describe the 3D ray tracing based simulator and the characterization tool used to extract the channel characteristic parameters.

2.1.3D Ray Tracing Simulator. In order to predict the wireless radio communication channel performances, the XLIMSIC laboratory has developed a radio propagation simulator $[28,36-38]$, based on a $3 \mathrm{D}$ ray tracing associated to the Geometrical Optics laws (GO) and the Uniform Theory of Diffraction (UTD). Input data are the environment modelled in three dimensions by facets (finite planes), the maximum number of electromagnetic interactions (reflection on facets, diffraction on dihedron, i.e., two facets with a common edge, and refraction through facets), in addition with antennas features of transmitters and receivers, studied frequency, and so forth. The output ones are complex impulse responses, received power, and Angles of Arrival and Departure (AoA and $\mathrm{AoD}$, resp.).

The $3 \mathrm{D}$ ray tracing method computes all the paths between receiver(s) and transmitter(s) for a fixed number of electromagnetic interactions. So this tool is based on path research technics ( $c f$. Figure 1) using the method of sources/images based on Snell's formalism to determine the reflected rays on flat surfaces in addition to the method of folding [39] based on the generalized Fermat's principle and allowing determining the diffracted rays on the edges. These technics are fast and simple to implement. Concerning the reflected rays ( $c f$. Figure $1(\mathrm{a})$ ), the reflection points $P_{1}$ and $P_{2}$ are computed from the symmetric (i.e., images) of the transmitter $T_{x}$ in relation to Wall 1 and Wall ${ }_{2}$. Concerning the diffraction rays (cf. Figure $1(\mathrm{~b})$ ), the diffracted point $\mathrm{D}$ is computed on the edge AB. The position of the diffraction point is known by its parameter $t$ as in (1), where $t_{T_{x}}$ (resp. $t_{R_{x}}$ ) denotes the parametric coordinate of the point $T_{p}$ (resp. $R_{p}$ ) which results of the projection of $T_{x}$ (resp. $R_{x}$ ) on the edge $\mathrm{AB}$, and where $d_{T_{x}}$ (resp. $d_{R_{x}}$ ) is the length of the vector $T_{x} T_{p}$ (resp. $R_{x} R_{p}$ )

$$
S=\frac{t_{T_{x}} \cdot d_{R_{x}}+t_{R_{x}} \cdot d_{T_{x}}}{d_{T_{x}}+d_{R_{x}}} .
$$

This simulator has been validated in the case of a rectangular straight tunnel in [40] by comparison with [41].

However, the complexity and the computation time of such a method grow in an exponential way with the number of facets composing the scatters, and the considered number of electromagnetic interactions. Indeed, being $N f$ the number of facets constituting the environment, $N_{a}$ the number 


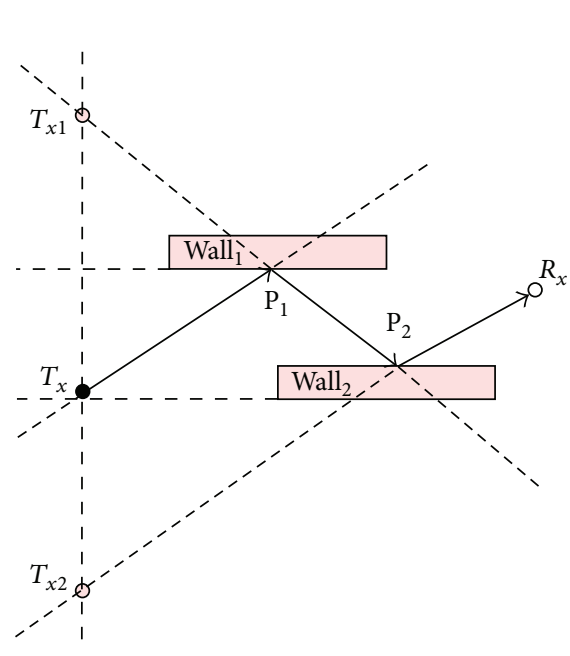

(a)

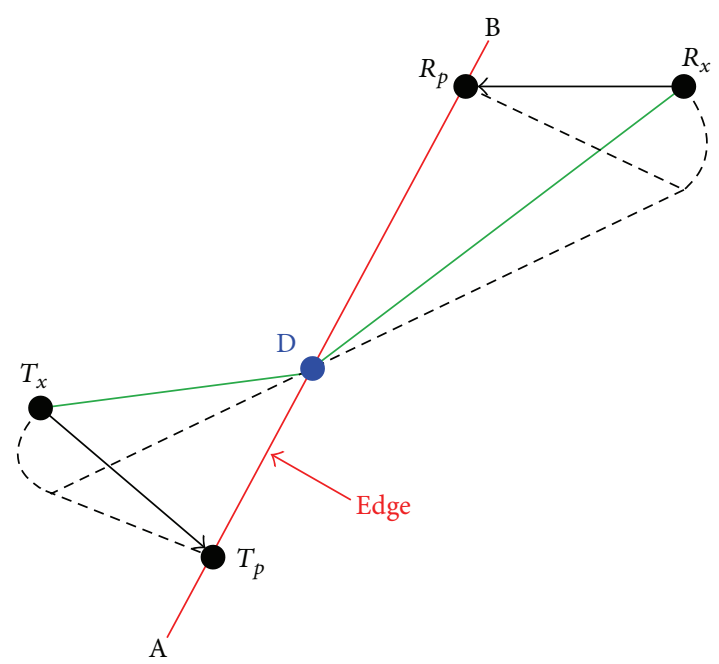

(b)

FIgURE 1: Path research technics: (a) source-image methods, (b) method of diffraction point computation.

of dihedral, $N_{r}$ and $N_{d}$, respectively, the number of considered reflections and diffractions, the number of potential paths $S$ between the transmitter and the receiver can be evaluated by

$$
S=C_{N_{d}}^{N_{r}+N_{d}} *(N f)^{N_{r}} *\left(N_{a}\right)^{N_{d}},
$$

where $C_{X}^{Y}$ is the binomial function.

As an example, the simulation of a static environment constituted of 1000 facets and 1500 dihedrals, considering a maximum of 3 reflections and 2 diffractions, leads to a number of potential paths equal to $2.25 * 10^{16}$. Furthermore, each potential path requires a calculation of the position of the reflection/diffraction points, besides the test of visibility for its physical validation. We propose in this paper to reduce the number of facets $N f$ (and so $N_{a}$ ) in order to minimize the simulator's computation time.

2.2. Channel Characterization Tool. In this subsection, we present the channel characterization tool also developed in the XLIM-SIC laboratory $[42,43]$. This tool calculates the various Fourier transforms of the Bello's system [44] to provide the various spectrums as the delays, the angles of arrival or the Doppler spectrums and their characteristic parameters such as delay spread, Doppler spread, time, and coherence bandwidth. The relevance of all the above parameters is subject to the assumption of Wide Sense Stationary Uncorrelated Scatters (WSSUSs). In other words, we consider that the 1st and 2nd order moments of the characteristic functions (impulse responses, frequency transfer function, etc.) are locally constant, inducing a stationary channel.

The channel parameters are calculated as follows.

(i) The received power is the result of the contribution of all $n$ received paths. The expression of this power is as follows:

$$
P=\left|\sum_{i=1}^{n} a_{i} \cdot e^{-j \varphi_{i}}\right|^{2},
$$

where $a_{i}$ and $\varphi_{i}$ are, respectively, the amplitude and phase of the considered path.

(ii) The power profiles or spectrum are functions that come from the Bello's system. They allow us to obtain information about the power associated with each path according to propagation delay, Doppler shift, arrival angles, and so forth. In the following, we describe some spectrum:

(i) Delay Spectrum

$$
S(\tau)=\sum_{i=1}^{n}\left|h\left(\tau, v_{i}\right)\right|^{2}
$$

(ii) Doppler Spectrum

$$
S(\nu)=\sum_{i=1}^{n}\left|h\left(\tau_{i}, v\right)\right|^{2},
$$

where $\tau$ and $\nu$ are the delay and Doppler frequency, respectively. From these spectra, we can calculate the mean delay, the mean Doppler, the delay, and Doppler spreads. The expressions are as follows:

(i) Mean delay and delay spread

$$
\begin{gathered}
\tau_{\text {mean }}=\frac{\sum_{\tau} \tau \cdot S(\tau)}{\sum_{\tau} S(\tau)}, \\
\sigma_{\tau}=\sqrt{\frac{\sum_{\tau}\left(\tau-\tau_{\text {mean }}\right)^{2} \cdot S(\tau)}{\sum_{\tau} S(\tau)}}
\end{gathered}
$$


(ii) Mean Doppler and Doppler spread

$$
\begin{gathered}
\nu_{\text {mean }}=\frac{\sum_{\nu} \nu \cdot S(\nu)}{\sum_{\nu} S(\nu)}, \\
\sigma_{\nu}=\sqrt{\frac{\sum_{\nu}\left(\nu-v_{\text {mean }}\right)^{2} \cdot S(\nu)}{\sum_{\nu} S(\nu)} .} .
\end{gathered}
$$

Note that to calculate the Doppler parameters above, we remain in its domain of validity $\left[-v_{\max }, v_{\max }\right]$, which is the maximum Doppler that depends on the mobile speed and the frequency used, whose expression is as follows:

$$
v_{\max }=\frac{V}{\lambda},
$$

where $V$ is the velocity of the moving $\left(\mathrm{m} \cdot \mathrm{s}^{-1}\right)$, and $\lambda$ is the wavelength (m).

In our study, we set a constant speed of $50 \mathrm{~km} \cdot \mathrm{h}^{-1}$ which is commonly used in subway applications.

\section{Identification Method of Nonsignificant Scatters}

Recall that the aim of this study is to simplify the geometrical description of the considered environment by removing all the facets that do not contribute enough to the energy balance, in order to accelerate the wave propagation simulations, as it was explained in Section 2.1, without compromising the accuracy of simulation results.

So we have in a first time to determine the impact of each environment facet on the wave propagation. According to the specific context of this paper (subway tunnel configuration), all facets will not be considered in a same manner. Indeed, as it is indicated in the literature, tunnel environments can be viewed as an oversized dielectric waveguide [5]. From this, the wave propagation mechanisms are very specific in this kind of environment. So to keep this specific behaviour, we exclude the possibility of eliminating facets constituting the guide, that is, the walls, roof, and ground of the tunnel. Furthermore, the guided propagation effect can be broken by the presence of a train parked inside the tunnel, while another train moves on another track. For this reason, we also exclude the possibility of eliminating facets constituting the parked train. To conclude and since our study focuses on dynamical scenario, we will be interested only in the facets of mobile trains.

Thus to identify the contribution degree of each facet to the energy balance, we retained as a criterion the main propagation parameter, to know the received power associated to the rays having interacted with this facet. Please notice that although the criterion of identification of the nonsignificant facets is the received power associated to this facet, the validation will be realized not only on the received power but also on the wide band characteristic parameters, such as delay spread or Doppler.

Now we have specified facets that can potentially be eliminated, and the first step of our method is to identify, at each position $i$ of a mobile train along its route, all the rays having interacted with the facets constituting this train. We can then compute Pref $_{i}$ which corresponds to the power associated to all of these rays, that is, the contribution of this train, and $\mathrm{Pf}_{i}$ which corresponds to the power associated to the facet $f$ constituting this train at the position $i$.

For each position $i$ of the train along its route in the tunnel, we calculate the instantaneous gap $\operatorname{errf}_{i}$ such as

$$
\operatorname{errf}_{i}=\left|\operatorname{Pref}_{i}-\operatorname{Pf}_{i}\right|
$$

From the computation of $\operatorname{errf}_{i}$ along the mobile train route, the contribution degree of the facet $f$ is obtained by application of the following criteria, based on two thresholds, noted $x_{1}$ and $x_{2}\left(x_{1}<x_{2}\right)$ such as the following:

(i) if $\operatorname{errf}_{i}$ is lower than the first threshold $x_{1}$ (in $\mathrm{dB}$ ), the facet $f$ belongs to the Contributive Facets category, noted CF, because the energy carried by rays having interacted with it mainly contribute to the one globally carried by the train facets;

(ii) if errf is upper than $x_{1}$ and lower than the second threshold $x_{2}$ (in $\mathrm{dB}$ ), the facet $f$ belongs to the category of Medium Contributive Facets; noted MCF;

(iii) if $\operatorname{errf}_{i}$ is upper than $x_{2}$, the facet $f$ belongs to the No Contributive Facet category, noted NCF, because the energy carried by rays having interacted with it is considered as nonsignificant compared to the one globally carried by the train facets.

Once a facet $f$ has been classified among the three previous categories (CF, MCF, and NCF) according to each position $i$ of the mobile train along its route in the tunnel, its global contribution degree corresponds to the one which presents the maximum occurrence over all positions along the mobile train route.

To implement this method, both thresholds $x_{1}$ and $x_{2}$ have naturally to be specified. In this way, we have chosen an empirical couple presenting an acceptable degree of accuracy for characteristic parameters computation: $x_{1}=10 \mathrm{~dB}$ and $x_{2}=20 \mathrm{~dB}$.

Indeed, for this couple of thresholds we consider a high margin of error $(10 \mathrm{~dB})$ to choose if a facet is contributive or not. So with these thresholds we have a pessimistic modelling of the environment, that is, lower limit of environment modelling simplification. Thus, to consider few values of threshold means to simplify more the environment.

Now we have presented the method allowing identifying the contribution degree of a facet; we will apply this last one on realistic scenarios to evaluate its performance in terms of accuracy.

\section{Implementation and Evaluation of the Method}

4.1. Scenario Description. In this scenario illustrated on Figure 1 we consider the case of a dynamical railway environment. We have modelled a two-track concrete tunnel $(\varepsilon r=$ $\left.10, \sigma=0.1 \mathrm{~S} \cdot \mathrm{m}^{-1}\right)$ that contains two metallic trains $(\varepsilon r=1$, 
$\left.\sigma=56000 \mathrm{~S} \cdot \mathrm{m}^{-1}\right)$. One of them is a fixed train parked on one track between 190 and $310 \mathrm{~m}$ that holds the receiver. The second train moves besides the second track from 500 to $-120 \mathrm{~m}$ with a step of $\lambda / 3$ ( $\lambda$ is the wavelength) based on the studies of Parson [45] and Sarkar et al. [46], that is, every $1.7 \mathrm{~cm}$ at the considered frequency of $5.8 \mathrm{GHz}$ related to the future communication systems [15], crossing the fixed train along its route. A small sampling step has been chosen in order to take into account all the variations of the received power that can appear from one position of the mobile train to another.

Furthermore, we have observed for this study that propagation channel is stationary (WSSUS), in terms of coherence time, on $10 \lambda$. This last point is in accordance with the studies of Parson who has concluded that on distance less than $48 \lambda$, the effects of slow and shadowing fading are unapparent [45].

The dimensions of the tunnel are $500 \times 4.5 \times 4.5 \mathrm{~m}^{3}$ and the ones of the two trains are $120 \times 3 \times 4 \mathrm{~m}^{3}$. The respective coordinates, $(x, y, z)$ in meter, of the fixed transmitter and receiver are $(8.8,500,4.1)$ and $(0.85,190,4.1)$. All antennas used here are vertical electrical dipoles and the transmitted power is equal to $1 \mathrm{~mW}$.

According to several previous studies carried out in railway environments [47], we have limited the number of reflections and diffractions to six and one, respectively, which is sufficient to guaranty the power convergence in our specific context of large and bi-ways tunnels $[16,48,49]$. Obviously no transmission is needed regarding the width of tunnel walls and the electrical mirror behaviour of the trains.

The facets are numbered from 0 to 5 as it is shown on Figure 2. Please notice that the facets 0 and 3 correspond to the train ground and roof, respectively.

\subsection{Identification of Nonsignificant Scatters and Simplified Model}

4.2.1. Global Study on the Complete Environment. We first apply the identification algorithm of nonsignificant scatters presented in Section 3 on the scenario of Figure 2 according to the couple of thresholds $\left(x_{1}=10 \mathrm{~dB}\right.$ and $x_{2}=20 \mathrm{~dB}$ ). Then, we trace on Figure 3, according to each facet of the mobile train, a histogram related to each category of facets (CF, MCF, and NCF). Each bar's height of the histogram is proportional to the occurrence of those resultant categories along the route of the mobile train. These values are normalized according to the number of train positions and so expressed in percentage of these positions.

From these histograms we can notice that the facets 4 and 0 , contrary to the others, are mainly in the NCF category $(60 \%$ and $10 \%$, resp.). Consequently we remove them to achieve the simplified model of Figure 4 according to the considered scenario.

We wish now to compare the performance between the simplified and the full models. Figure 5 shows the evolution of the total received power respectively obtained from the full model and the simplified one.
Figure 5 shows a significant degradation of the prediction in a zone going from 250 to $350 \mathrm{~m}$. This phenomenon is going to be explained in the next paragraph.

This studied scenario is very interesting because of the location of the receiving antenna. Indeed, the mobile train firstly moves in the direction of the receiver, that is, fixed in the middle of the tunnel and then goes away from it. It means that some facets will be or not be visible from the receiver depending on the location of the mobile train along the tunnel. The mobile train is initially located at the tunnel entrance. At this location, only facets 3 and 4 are visible from both the transmitter and the receiver. When the mobile train enters completely in the tunnel, facet 5 becomes visible from the transmitter. Facet 4 becomes not visible from the transmitter but is still visible from the receiver. Except facet 0 (the mobile train ground), all facets of the mobile train are visible from at least one antenna. When the mobile train exceeds the fixed one, facet 4 becomes hidden from both the transmitter and the receiver. Thus we identify three zones.

(i) The first one goes from 500 to $380 \mathrm{~m}$ and corresponds to the zone where only facets 1 and 3 are visible from the transmitter, and where facets 2,3 , and 4 are visible from the receiver. Facets 0 and 5 are hidden. In this configuration, one facet (number 3 ) is directly visible from the two antennas.

(ii) The second one goes from 380 to $190 \mathrm{~m}$, where we have noticed the main difference between the curves obtained from each model ( $c f$. Figure 5), and corresponds to the zone where facets 1,3 , and 5 of the mobile train are visible from the transmitter, while facets 2,3 , and 4 are visible from the receiver. Only facet 0 is hidden. In this configuration, only facet 3 is visible from both the transmitter and the receiver.

(iii) Finally the third zone goes from 190 to $-120 \mathrm{~m}$ and corresponds to the zone where facets $1,2,3$, and 5 are visible from the receiver and/or from the transmitter. Facets 4 and 0 are thus hidden. One more time, there is one facet visible from the two antennas, facet 3.

4.2.2. Study by Zone. From these last remarks, we imply the identification algorithm of nonsignificant scatters ( $c f$. Section 3) on each zone previously identified.

First Zone: From 500 to $380 \mathrm{~m}$. The histograms of Figure 6(a) show that the most contributive facet is facet 3 . Facets 4,2 , and 1 mainly present contributive or medium contributive values. Finally facets 0 and 5 are always noncontributive.

Thus we propose to only model facet 1,2,3, and 4 in the first zone.

Second Zone: From 380 to $190 \mathrm{~m}$. The analysis of Figure 6(b) indicates that in the second zone, all the facets of the mobile train are mainly contributive (CF or MCF category) except facet 0 which is always noncontributive. 


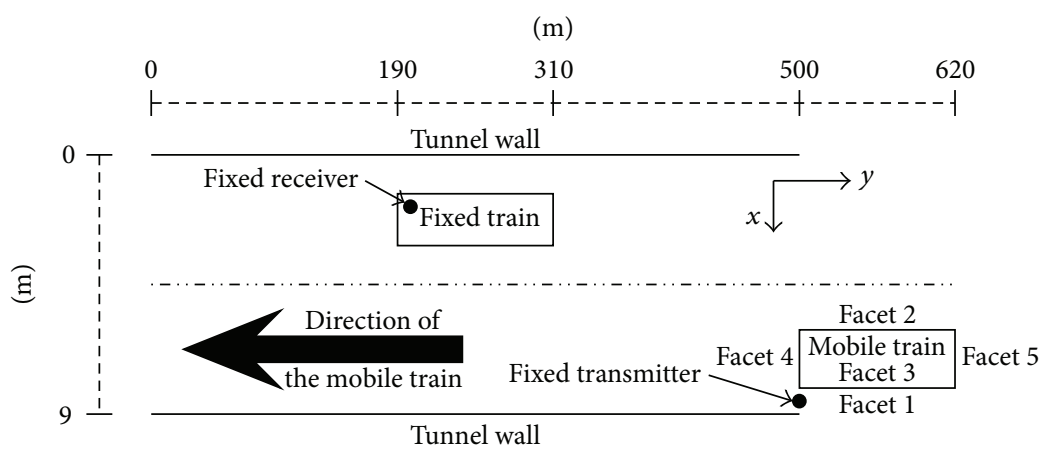

FIGURE 2: Description of the scenario.

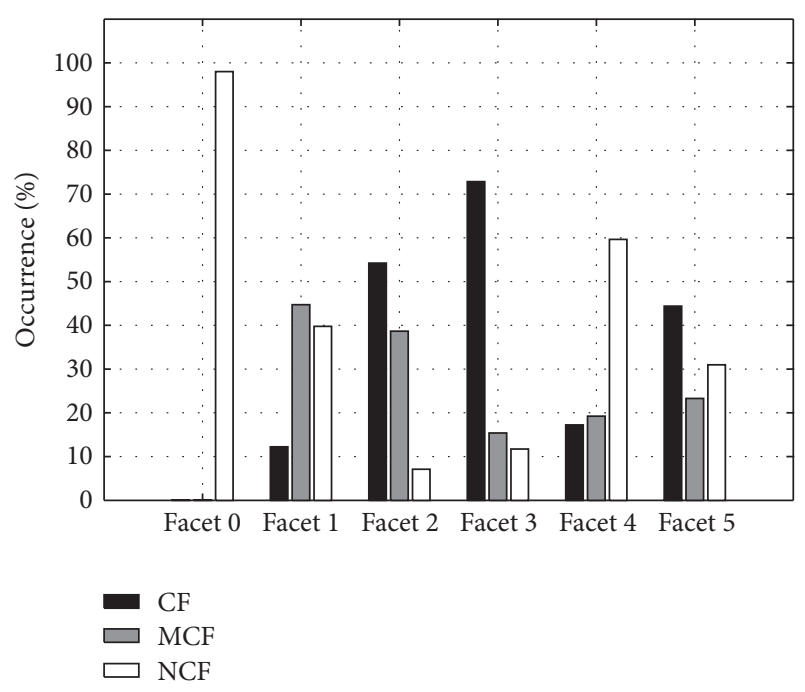

FIGURE 3: Identification of contribution degree of the facets along the mobile train route.

So we propose to only remove facet 0 in the second zone.

Third Zone: From 190 to $-120 \mathrm{~m}$. Finally in the third zone, the histograms of Figure 6(c) show that only facets 0 and 4 are mainly non-contributive.

So in zone 3 , facets $1,2,3$, and 5 compose the proposed simplified model.

Synthesis: Definition of a Simplified Model Composed of 3 Zones. From a geometrical analysis of the second scenario, we define a new simplified model that depends on the location of the mobile train. In the first zone (from 500 to $380 \mathrm{~m}$ ) we only model facets $1,2,3$, and 4 . In the second zone, we only model facets $1,2,3,4$, and 5. Finally in the third zone, the modelled facets are facets $1,2,3$, and 5 . It is interesting to notice that the modelled facets are those that are visible at least from an antenna.

To evaluate this simplified model, we compare in the next section the narrow and wide-band channel parameters obtained from this model to those ones obtained from the full model (all the mobile train facets).
TABLE 1: Mean and standard deviation of the absolute errors of the considered characteristic parameters.

\begin{tabular}{lcc}
\hline & $\begin{array}{c}\text { Mean absolute } \\
\text { error }\end{array}$ & $\begin{array}{c}\text { Standard deviation } \\
\text { absolute error }\end{array}$ \\
\hline Mean Delay (ns) & 0.032 & 0.104 \\
Delay spread (ns) & 0.026 & 0.076 \\
Mean Doppler (Hz) & 0.001 & 0.003 \\
Doppler spread (Hz) & 0.041 & 0.324 \\
\hline
\end{tabular}

\subsubsection{Validation on Narrow and Wide-Band Results}

Received Power. Figure 7 presents the comparison between the total received power along the mobile train route according to the full model and the simplified one per zone, respectively. It shows that the two curves are very close to each other.

This observation is comforted by the mean and standard deviation values of the absolute error between the both models. They are, respectively, equal to $0.18 \mathrm{~dB}$ and $0.47 \mathrm{~dB}$.

Wide-Band Parameters. To evaluate the performances of our simplified model, we have computed the wide-band parameters associated to this one and compared them to those obtained from the full model. Table 1 summarizes these results in terms of mean error and standard deviation of the error according to mean delay, delay spread, mean Doppler, and Doppler spread. All the values are very weak.

\section{Engineering Method to Simplify the Environments Description}

5.1. Introduction. From the analysis of the previous scenario, we have shown that it is possible to simplify the geometrical description of the environment without degrading the results accuracy. In this way we have applied our identification algorithm of nonsignificant scatters and we have connected the simplification of the environment to the identification of several zones of the tunnel in which the facets have specific visibility relations with the antennas. Indeed, one 


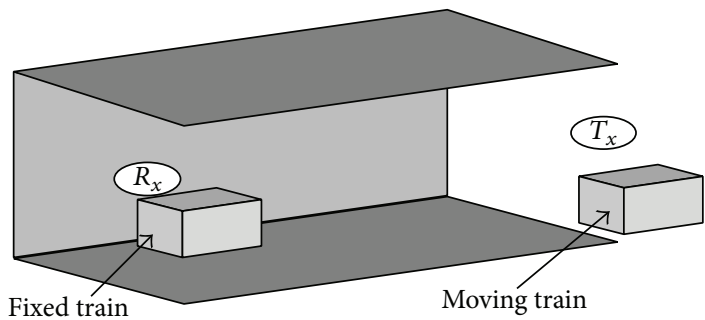

(a)

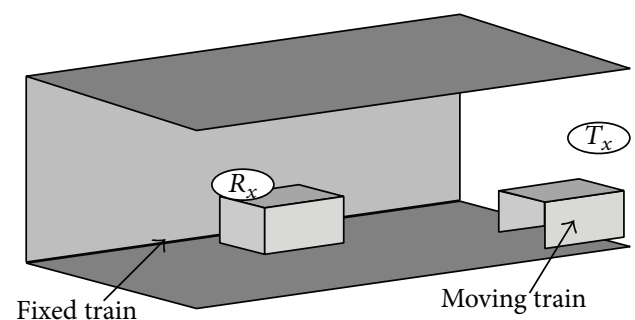

(b)

FIGURE 4: Modelling of propagation environment: (a) the full model, (b) the simplified model.



FIGURE 5: Evolution of the received power along the mobile train route according to the full model and the simplified model.

can distinguish three cases: the facets visible from both the transmitter and the receiver (i.e., CF category), the facets visible from the transmitter or the receiver only (MCF category), and the facets hidden from both the transmitter and the receiver (NCF category).

So we propose an engineering method consisting in removing the facets of NCF category on these visibility criteria, without applying our identification algorithm of nonsignificant scatters. In the next section we evaluate this approach on a last complex scenario.

5.2. Presentation of a Complex Scenario. Figure 8 illustrates this last complex scenario considering a $4 \times 4 \mathrm{MIMO}$ channel. It is composed of a two-track tunnel in which two trains move in opposite directions and cross themselves. The tunnel dimensions are the same that for the previous scenarios. To minimize the correlation, the spacing between transmitters or receivers antennas is equal to $50 \mathrm{~cm}$, that is, $10 \lambda$. The nearer transmitter is fixed at $(0.75,0,4.3)$ on the top side of the tunnel, while the receiver is fixed on the front of the train number 1 that moves on the same track.
To simplify this environment description, we propose to apply the geometrical criteria presented in Section 5.1. One more time this one drives to consider three different zones in the tunnel:

(i) zone 1 , from -1 to $120 \mathrm{~m}$ : only facets 3 , 9, and 10 are visible both from the transmitting and receiving antennas. Furthermore facets 4 and 6 are only visible from the transmitters and the receivers, respectively;

(ii) zone 2, from 120 to $250 \mathrm{~m}$ : facet 8 becomes visible from the transceivers;

(iii) zone 3, from 250 to $500 \mathrm{~m}$ : facets 8 , 9, and 10 are visible both from the transmitters and receivers.

5.3. Validation. So due to the previous analyses, we propose to model the mobile train number one by their facets 3 and 4 whatever the considered zones. The other mobile train is modelled by facets 6,9 , and 10 in the zone 1 ; by facets $6,8,9$, and 10 in the zone 2 ; and by facets 8,9 , and 10 in the last zone (cf. Figure 9). Table 2 summarizes the modelling.

In the following, we compare the received power, delay spread, mean delay, Doppler spread, and mean Doppler obtained from the complete and the simplified modelling. We note, as it is shown in the Figure 10 and Table 3, that the results are quasi identical.

Finally, we compare the computation time between the full model and the simplified one obtained using a $2 \mathrm{GHz}$ mono core processor. Recall that, due to the considered trainmoving step $(1.7 \mathrm{~cm})$, each previously presented scenario drives to about 30,000 launches of the ray-tracing routine. Furthermore, by applying (2) modelling the complexity of the simulation, we obtain about $10^{9}$ potential paths for the full model and about $10^{5}$ for the simplified one, so a gain equal to $10^{4}$. Nevertheless, this reduction of potential paths does not lead to a similar gain in computation time because of the informatics implementation, data storage and so on.

Consequently, the simulation of the full model drives to a computation time of $54 \mathrm{~d} 3 \mathrm{~h} 57 \mathrm{~min}$ while the one related to the simplified model equals to $8 \mathrm{~d} 9 \mathrm{~h} 17 \mathrm{~min}$. So the reduction factor of the computation time is equal to 6 . 


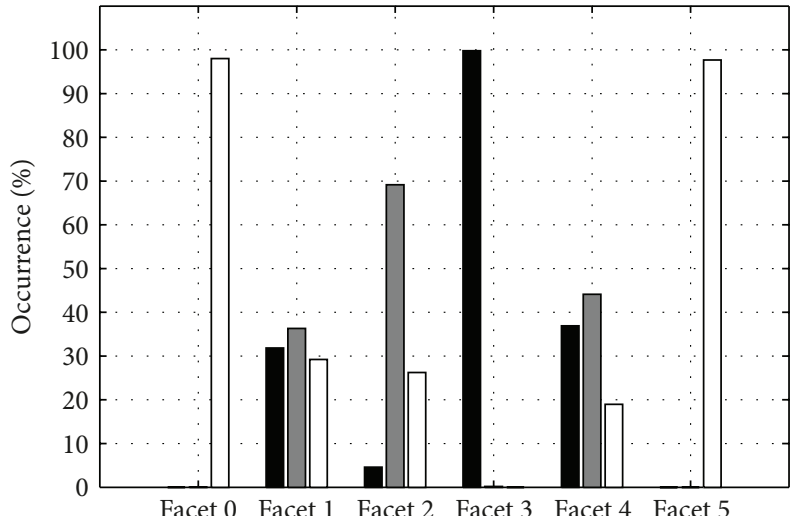

Facet 0 Facet 1 Facet 2 Facet 3 Facet 4 Facet 5
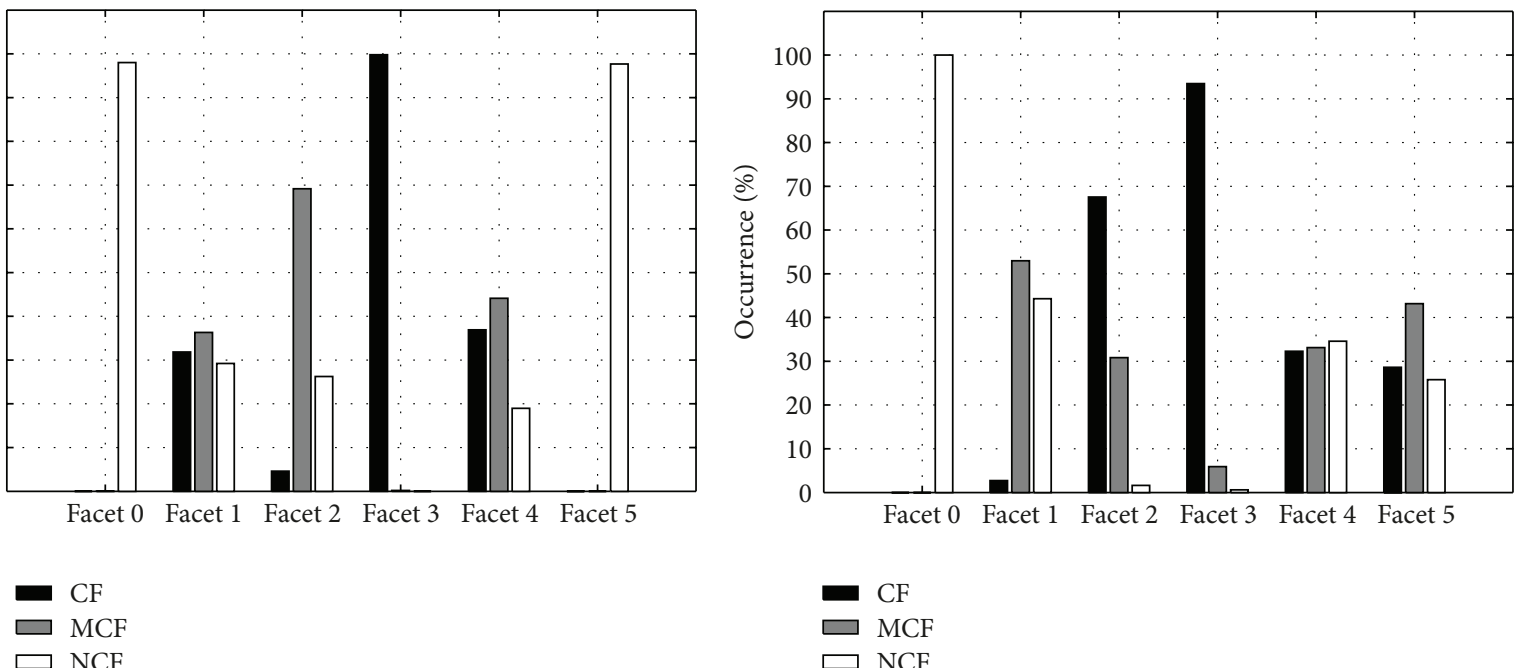

$\square \mathrm{MCF}$

$\square \mathrm{NCF}$

(a)

(b)

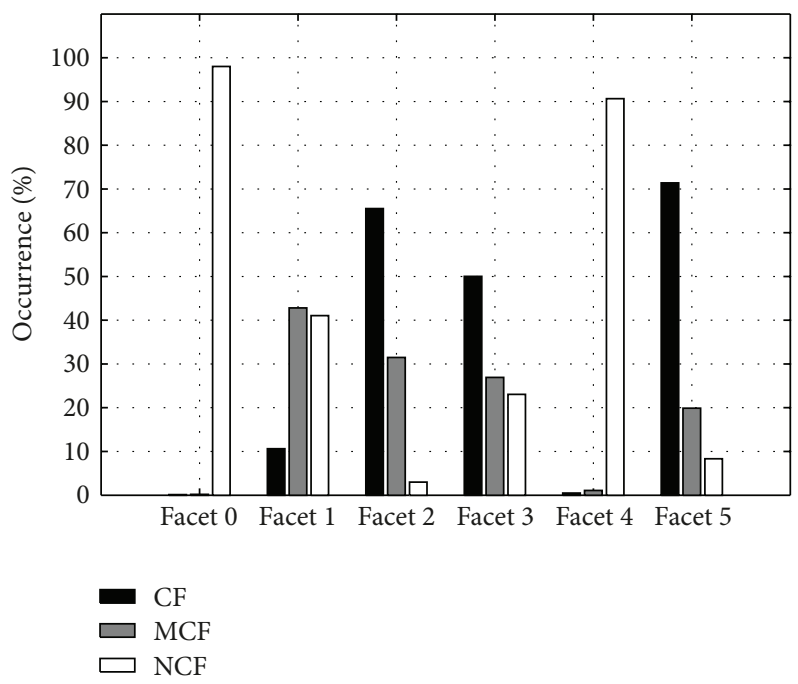

(c)

FIGURE 6: Identification of contribution degree of the facets in (a) the first zone, (b) the second zone, and (c) the third zone.

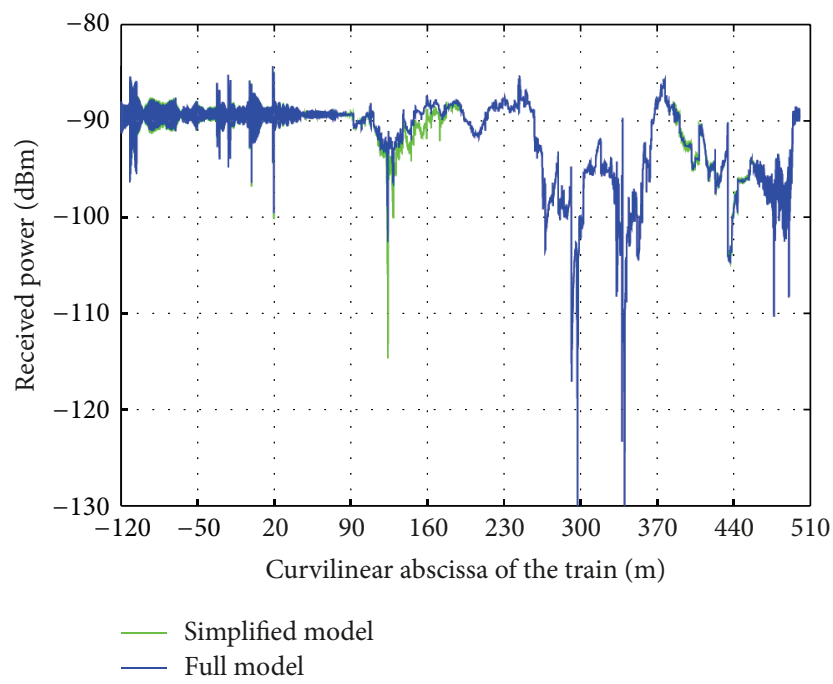

FIGURE 7: Evolution of the received power along the mobile train route according to the full model and the simplified one per zone. 
(m)

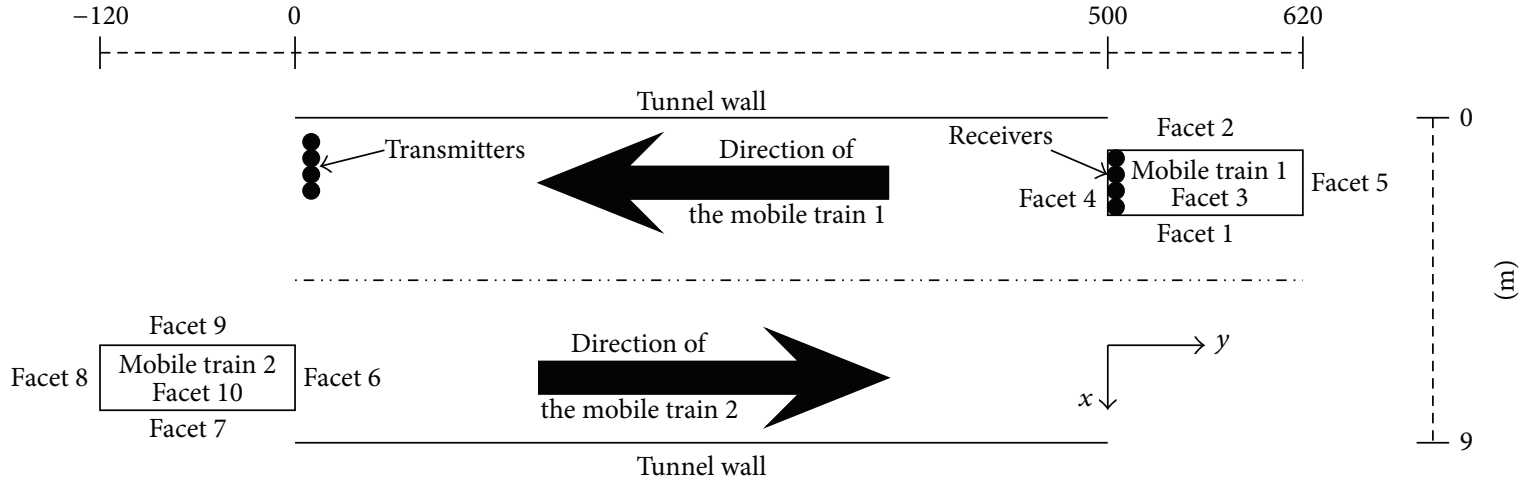

Figure 8: Description of a complex scenario.

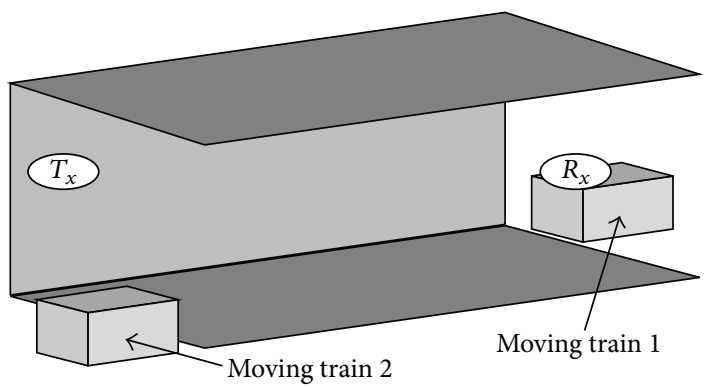

(a)

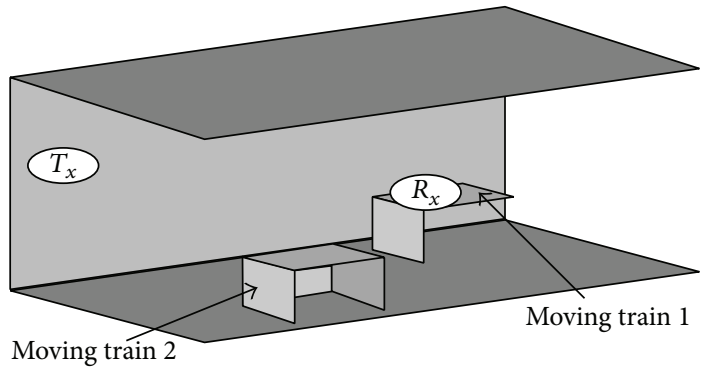

(c)

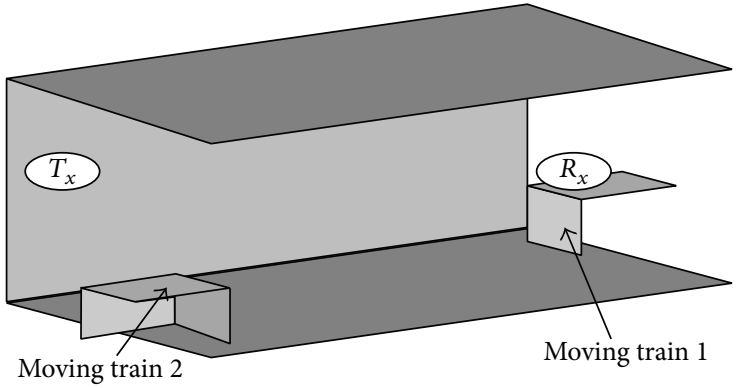

(b)

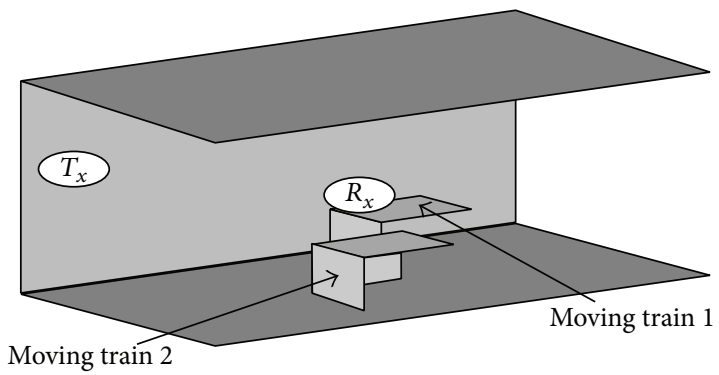

(d)

FIGURE 9: Modelling of propagation environment: (a) the full model, (b) the simplified model in zone 1, (c) the simplified model in zone 2 , and (d) the simplified model in zone 3 .

TABLE 2: Trains modelling per zone.

\begin{tabular}{lccc}
\hline Considering facets & Zone 1 & Zone 2 & Zone 3 \\
\hline Mobile train 1 & 3 and 4 & 3 and 4 & 3 and 4 \\
Mobile train 2 & 6, 9, and 10 & 6, 8, 9, and 10 & 8, 9, and 10 \\
\hline
\end{tabular}

\section{Conclusion}

This investigation allowed us to reduce the simulation time of a dynamical environment associated with a $4 \times 4 \mathrm{MIMO}$ channel at a frequency of $5.8 \mathrm{GHz}$.

For this, we have implemented an algorithm that allows identifying the contribution degree of the facets composing the mobile railway environment. This last one has led to the elaboration and then to the validation of a new algorithm
TABLE 3: Mean and standard deviation of the absolute errors of the considered characteristic parameters.

\begin{tabular}{lccc}
\hline & $\begin{array}{c}\text { Mean } \\
\text { reference } \\
\text { values (full } \\
\text { model) }\end{array}$ & $\begin{array}{c}\text { Mean } \\
\text { absolute error }\end{array}$ & $\begin{array}{c}\text { Standard } \\
\text { deviation } \\
\text { absolute } \\
\text { error }\end{array}$ \\
\hline Received power (dBm) & -80 & 1.2 & 2.3 \\
Mean Delay (ns) & 2100 & 71.9 & 71.3 \\
Delay spread (ns) & 600 & 53.9 & 59.9 \\
Mean Doppler (Hz) & 240 & 2.1 & 2.3 \\
Doppler spread (Hz) & 75 & 3.6 & 3.9 \\
\hline
\end{tabular}

based on simple geometrical criteria. These criteria are related to visibility relations between the antennas and the mobile 


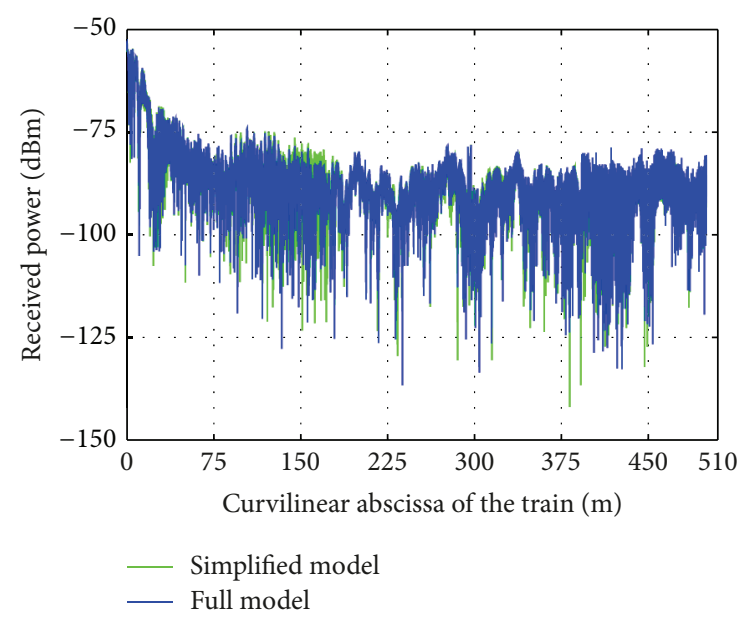

FIGURE 10: Evolution of the received power along the mobile train route according to the full and simplified models.

facets. This algorithm being applied before the ray-tracing routine, it is complementary to existing other acceleration methods previously published.

The obtained results are potentially interesting since we have shown that it is possible to divide the computation time by about 6 without compromising the prediction accuracy of the channel characteristics parameters such as delay or Doppler spreads.

\section{Acknowledgments}

The authors thank Mr. Jean-François CAILBAULT for developing the tool used to extract the wide-band parameters and the database to stock the obtained results. Moreover, these works have been supported by the ANR PREDIT MOCAMIMODYN project.

\section{References}

[1] M. Liénard, P. Degauque, and J. M. Molina-Garcia-Pardo, "Wave propagation in tunnels in a MIMO context-a theoretical and experimental study," Comptes Rendus Physique, vol. 7, no. 7, pp. 726-734, 2006.

[2] Y. Cocheril, C. Langlais, M. Berbineau, and G. Moniak, "Advantages of simple MIMO schemes for robust or high data rate transmission systems in underground tunnels," in Proceedings of the 68th IEEE Vehicular Technology Conference (VTC'08), pp. 1-5, Calgary, Canada, September 2008.

[3] T. Klemenschits and E. Bonek, "Radio coverage of tunnels by discret antennas: measurements and predictions," COST 231 TD(95031), 1995.

[4] M. Lienard and P. Degauque, "Propagation in wide tunnels at 2 GHZ: a statistical analysis," IEEE Transactions on Vehicular Technology, vol. 47, no. 4, pp. 1322-1328, 1998.

[5] Y. P. Zhang, "Novel model for propagation loss prediction in tunnels," IEEE Transactions on Vehicular Technology, vol. 52, no. 5, pp. 1308-1314, 2003.

[6] H. W. Chang, Y. H. Wu, S. M. Lu, W. C. Cheng, and M. H. Sheng, "Field analysis of dielectric waveguide devices based on coupled transverse-mode integral equation-numerical investigation," Progress in Electromagnetics Research, vol. 97, pp. 159-176, 2009.

[7] S. Y. Reutskiy, "The methods of external excitation for analysis of arbitrarily-shaped hollow conducting waveguides," Progress in Electromagnetics Research, vol. 82, pp. 203-226, 2008.

[8] P. Bernardi, D. Caratelli, R. Cicchetti, V. Schena, and O. Testa, "A numerical scheme for the solution of the vector parabolic equation governing the radio wave propagation in straight and curved rectangular tunnels," IEEE Transactions on Antennas and Propagation, vol. 57, no. 10, pp. 3249-3257, 2009.

[9] A. V. Popov and N. Y. Zhu, "Modeling radio wave propagation in tunnels with a vectorial parabolic equation," IEEE Transactions on Antennas and Propagation, vol. 48, no. 9, pp. 1403-1412, 2000.

[10] K. D. Laakmann and W. H. Steier, "Waveguides: characteristic modes of hallow rectangular dielectric waveguides," Applied Optics, vol. 15, no. 5, pp. 1334-1340, 1976.

[11] A. G. Emslie, R. L. Lagace, and P. F. Strong, "Theory of the propagation of UHF radio waves in coal mine tunnels," IEEE Transactions on Antennas and Propagation, vol. 23, no. 2, pp. 192-205, 1975.

[12] I. F. Akyildiz, Z. Sun, and M. C. Vuran, "Signal propagation technics for wireless underground communication networks," Physical Communication, vol. 2, no. 3, pp. 167-183, 2009.

[13] D. G. Dudley and S. F. Mahmoud, "Linear source in a circular tunnel," IEEE Transactions on Antennas and Propagation, vol. 54, no. 7, pp. 2034-2047, 2006.

[14] D. Dudley, M. Lienard, S. Mahmoud, and P. Degauque, "Wireless propagation in tunnels," IEEE Transactions on Antennas and Propagation, vol. 49, no. 2, pp. 11-26, 2009.

[15] K. Guan, Z. Zhong, R. He, Y. Li, and C. B. Rodriguez, "Propagation mechanism modeling in the near-region of arbitrary cross-section tunnels," International Journal on Antennas and Propagation, vol. 2012, Article ID 183145, 11 pages, 2012.

[16] P. Mariage, M. Lienard, and P. Degauque, "Theoretical and experimental approach of the propagation of high frequency waves in road tunnels," IEEE Transactions on Antennas and Propagation, vol. 42, no. 1, pp. 75-81, 1994.

[17] T. S. Wang and C. F. Yang, "Simulations and measurements of wave propagations in curved road tunnels for signals from GSM base stations," IEEE Transactions on Antennas and Propagation, vol. 54, no. 9, pp. 2577-2584, 2006.

[18] D. Didascalou, J. Maurer, and W. Wiesbeck, "Subway tunnel guided electromagnetic wave propagation at mobile communications frequencies," IEEE Transactions on Antennas and Propagation, vol. 49, no. 11, pp. 1590-1596, 2001.

[19] B. Ai, R. He, Z. Zhong et al., "Radio wave propagation scene partitioning for high-speed rails," International Journal on Antennas and Propagation, vol. 2012, Article ID 815332, 7 pages, 2012.

[20] J. Maurer, T. Fugen, M. Porebska, T. Zwick, and W. Wiesbeck, "Ray optical channel model for mobile to mobile communications," COST, 2100 TD(08430), 2008.

[21] J. Maurer, T. Fugen, T. Schafer, and W. Wiesbeck, "A new intervehicle communications (IVC) channel model," in Proceedings of the IEEE Vehicular Technology Conference (VTC Fall '04), Los Angeles, Calif, USA, September 2004.

[22] J. Maurer, T. Fugen, and W. Wiesbeck, "Narrow-band measurement and analysis of the inter-vehicle transmission channel at $5.2 \mathrm{GHz}$," in Proceedings of the IEEE Vehicular Technology Conference (VTC Spring '02), Birmingham, Ala, USA, 2002. 
[23] J. P. Rossi and Y. Gabillet, "A mixed ray launching/tracing method for full 3-D UHF propagation modeling and comparison with wide-band measurements," IEEE Transactions on Antennas and Propagation, vol. 50, no. 4, pp. 517-523, 2002.

[24] T. Rautiainen, R. Hoppe, and G. Wölfle, "Measurements and 3D ray tracing propagation predictions of channel characteristics in indoor environments," in Proceedings of the 18th Annual IEEE International Symposium on Personal, Indoor and Mobile Radio Communications (PIMRC '07), Athens, Greece, September 2007.

[25] H. W. Son and N. H. Myung, "A new approach to 3-D ray tracing for a microcellular propagation prediction model in urban environments," Microwave and Optical Technology Letters, vol. 23, no. 3, pp. 159-163, 1999.

[26] G. E. Athanasiadou, A. R. Nix, and J. P. McGeehan, "Ray tracing algorithm for microcellular wideband propagation modelling," in Proceedings of the IEEE 45th Vehicular Technology Conference (VTC '95), pp. 261-265, Chicago, III, USA, July 1995.

[27] T. Imai, M. Sumi, and T. Taga, "Propagation prediction system for Urban Area Macrocells using ray-tracing methods," NTT DoCoMo Technical Journal, vol. 6, no. 1, 2000.

[28] E. Masson, P. Combeau, Y. Cocheril, M. Berbineau, L. Aveneau, and R. Vauzelle, "Radio wave propagation in arch-shaped tunnels: measurements and simulations by asymptotic methods," Comptes Rendus Physique, vol. 11, no. 1, pp. 44-53, 2010.

[29] G. Wolfle, R. Hope, and F. M. Landstorfer, "A fast and enhanced ray optical propagation model for indoor and urban scenarios, based on an intelligent preprocessing of the database," in Proceedings of the IEEE International Symposium on Personal Indoor and Mobile Radio Communications (PIMRC '99), Osaka, Japan, September 1999.

[30] R. Hoppe, P. Wertz, F. M. Landstorfer, and G. Wölfle, "Advanced ray-optical wave propagation modelling for urban and indoor scenarios including wideband properties," European Transactions on Telecommunications, vol. 14, no. 1, pp. 61-69, 2003.

[31] T. Imai, "Novel ray-tracing acceleration technique using genetic algorithm for radio propagation prediction," NTT Technical Review, vol. 6, no. 2, 2008.

[32] M. Shevtsov, A. Soupikov, and A. Kapustin, "Highly parallel fast KD-tree construction for interactive ray tracing of dynamic scenes," Computer Graphics Forum, vol. 26, no. 3, pp. 395-404, 2007.

[33] D. Kopta, A. Kensler, T. Ize, J. Spjut, E. Brunvand, and A. Davis, "Fast effective bvh updates for dynamic ray-traced scenes using tree rotations," Tech. Rep. UUCS 11-002, University of Utah, 2011.

[34] F. A. Agelet, F. P. Fontán, and A. Formella, "Fast ray tracing for microcellular and indoor environments," IEEE Transactions on Magnetics, vol. 33, no. 2, pp. 1484-1487, 1997.

[35] M. Kimpe, H. Leib, O. Maquelin, and T. H. Szymanski, "Fast computational techniques for indoor radio channel estimation," IEEE Computing in Science \& Engineering, vol. 1, no. 1, pp. 31-41, 1999.

[36] F. Escarieu, Y. Pousset, L. Aveneau, and R. Vauzelle, "Outdoor and indoor channel characterization by a $3 \mathrm{D}$ simulation software," in Proceedings of the 12th International Symposium on Personal, Indoor and Mobile Radio Communications (PIMRC '01), pp. B105-B111, Boston, Mass, USA, October 2001.

[37] F. Mora and L. Aveneau, Optimised Scanning of a Visibility Graph Data Structure for Efficient Ray-Tracing, European Centre for Women and Technology, Paris, France, 2005.

[38] P. Combeau, L. Aveneau, R. Vauzelle, and Y. Pousset, "Efficient 2-D ray-tracing method for narrow and wideband channel characterisation in microcellular configurations," IEE Proceedings Microwaves, Antennas and Propagation, vol. 153, no. 6, pp. 502-509, 2006.

[39] L. Aveneau, Y. Pousset, R. Vauzelle, and M. Mériaux, "Development and evaluations of physical and computer optimizations for the UTD 3d model," in Proceedings of the Millennium Conference on Antennas \& Propagation (AP '00), Davos, Switzerland, April 2000.

[40] E. Masson, P. Combeau, M. Berbineau, R. Vauzelle, and Y. Pousset, "Radio wave propagation in arched cross section tunnelssimulations and measurements," Journal of Communications, vol. 4, no. 4, pp. 276-283, 2009.

[41] S. H. Chen and S. K. Jeng, "SBR image approach for radio wave propagation in tunnels with and without traffic," IEEE Transactions on Vehicular Technology, vol. 45, no. 3, pp. 570578, 1996.

[42] Y. Chartois, Y. Pousset, and R. Vauzelle, "A spatio-temporal radio channel characterization with a $3 \mathrm{D}$ ray tracing propagation model in urban environment," in Proceedings of the IEEE 15th International Symposium on Personal, Indoor and Mobile Radio Communications (PIMRC '04), pp. 2431-2435, Barcelona, Spain, September 2004.

[43] P. Carlos, P. Yannis, V. Rodolphe, and C. Pierre, "Sensitivity of the MIMO channel characterization to the modeling of the environment," IEEE Transactions on Antennas and Propagation, vol. 57, no. 4, pp. 1218-1227, 2009.

[44] P. A. Bello, "Characterization of randomly time-variant linear channels," IEEE Transactions on Communications, vol. 11, no. 4, pp. 360-393, 1963.

[45] D. Parson, The Mobile Radio Propagation Channel, WileyPentech, 1992.

[46] T. K. Sarkar, Z. Ji, K. Kim, A. Medouri, and M. SalazarPalma, "A survey of various propagation models for mobile communication," IEEE Antennas and Propagation Magazine, vol. 45, no. 3, pp. 51-82, 2003.

[47] D. J. Cichon, T. C. Becker, and W. Wiesbeck, "Determination of time-variant radio links in high-speed train tunnels by ray optical modeling," in Proceedings of the IEEE Antennas and Propagation Society International Symposium, pp. 508-511, June 1995.

[48] F. Fushini and G. Falciasecca, "A mixed rays-modes approach to the propagation in real road and railway tunnels," IEEE Transactions on Antennas and Propagation, vol. 2, no. 60, pp. 508-511, 2012.

[49] A. Hrovat, G. Kandus, and T. Javornik, "Impact of tunnel geometry and its dimensions on path loss at UHF frequency band," in Proceedings of the International Conference on Circuits, Systems, Communications \& Computers (SysCon '11), pp. 253258, Montreal, Canada, 2011. 



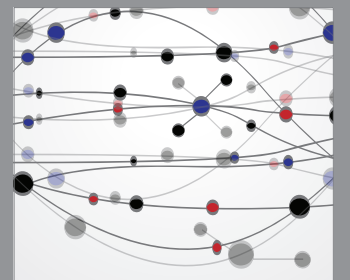

The Scientific World Journal
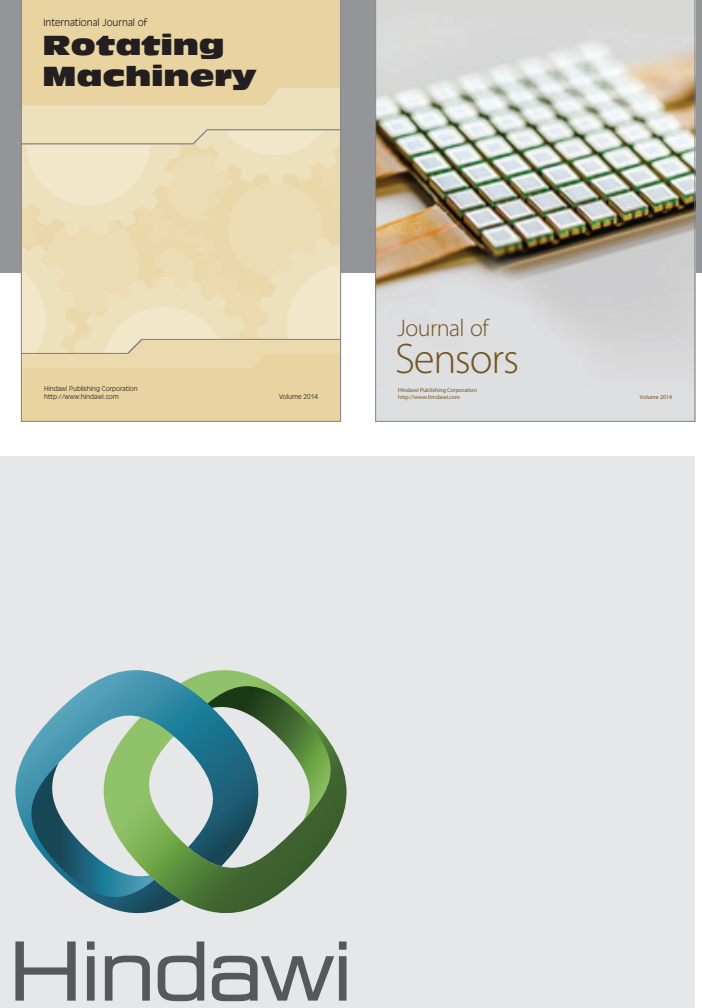

Submit your manuscripts at http://www.hindawi.com

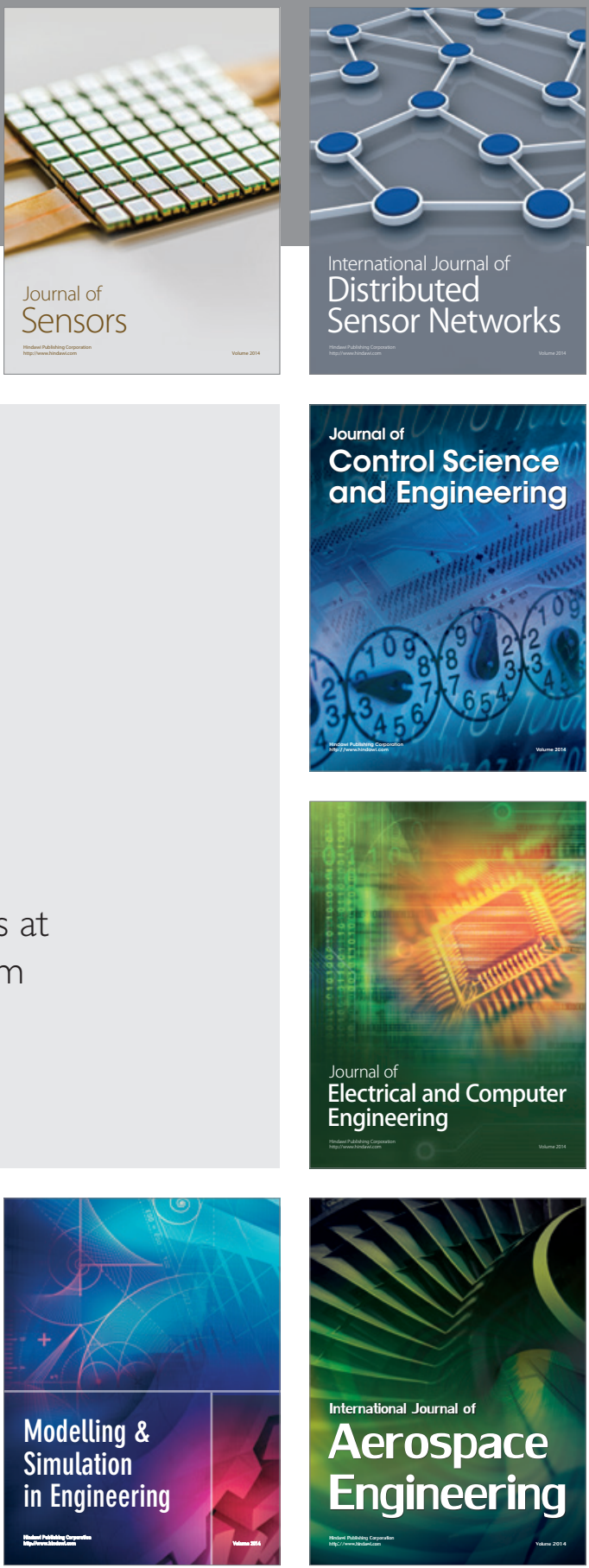

Journal of

Control Science

and Engineering
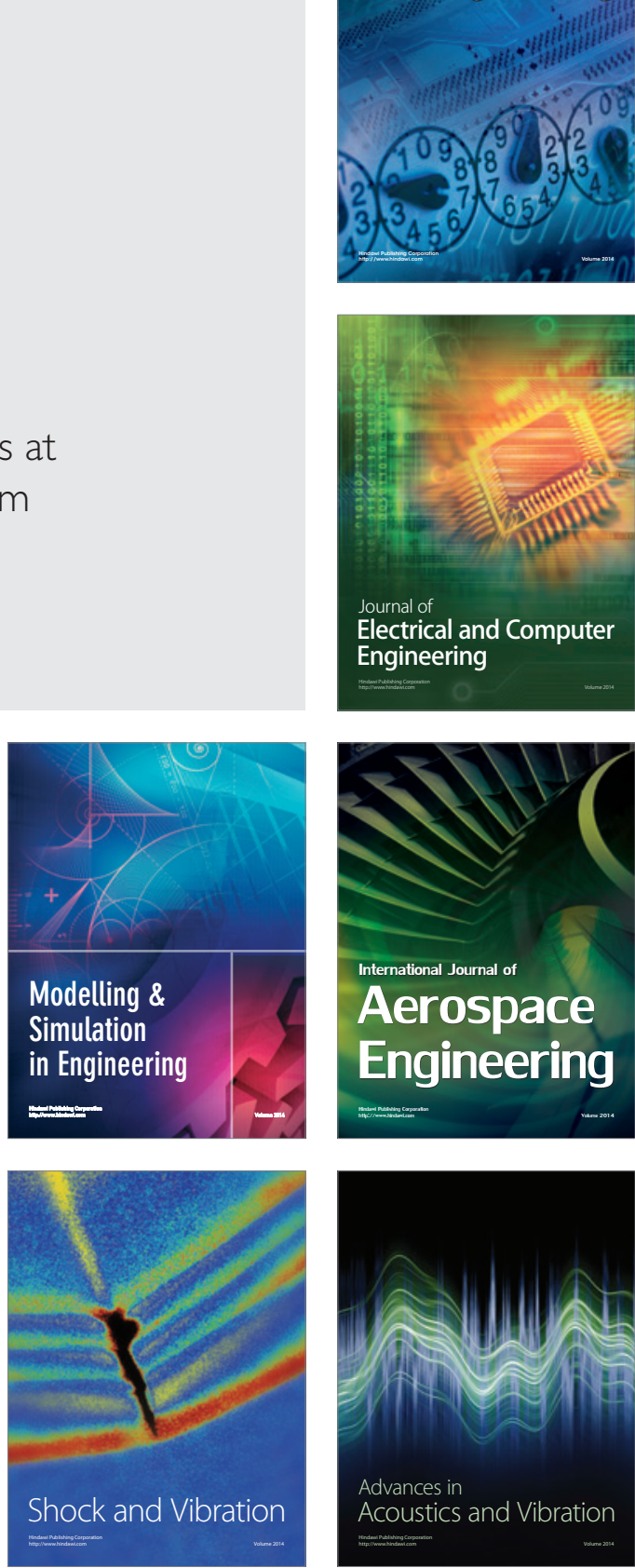